\title{
CONIFOLDS FROM D-BRANES
}

\author{
SUBIR MUKHOPADHYAY AND KOUSHIK RAY
}

\begin{abstract}
In this note we study the resolution of conifold singularity by D-branes by considering compactification of D-branes on $\mathbb{C}^{3} /\left(\mathbb{Z}_{2} \times \mathbb{Z}_{2}\right)$. The resulting vacuum moduli space of D-branes is a toric variety which turns out to be a resolved conifold, that is a nodal variety in $\mathbb{C}^{4}$. This has the implication that all the corresponding phases of Type-II string theory are geometrical and are accessible to the D-branes, since they are related by flops.
\end{abstract}

In the aftermath of the second superstring revolution the role of D-branes in the study of space-time has been widely appreciated. It has become quite apparent that one can probe sub-stringy scales in spacetime using D-branes - the BPS states that carry Rammond-Rammond charges in string theory. D-branes have recently been used to understand the short-distance geometry of Calabi-Yau manifolds [1] 3]. These studies discern rather directly, as compared to the studies with fundamental strings, the phase structure of Calabi-Yau manifolds [1,33]. It has been shown that the topological and geometric properties of Calabi-Yau manifolds studied using both D-branes and fundamental strings corroborate. More specifically, it has been shown [3] that the D0-branes do not allow for non-geometric phases to appear in the theory which is in keeping with the conclusion drawn earlier from the analysis of M-theory and F-theory [4].

The analysis of [3] was for $\mathbb{C}^{3} / \Gamma$, where $\Gamma$ is a discrete Abelian group in $S U(3)$. Specific examples of $\Gamma=\mathbb{Z}_{3}$ and $\Gamma=\mathbb{Z}_{5}$ were worked out, which could be generalized for any $\mathbb{Z}_{n}$. It was concluded that the Dbranes probe resolved $\mathbb{C}^{3} / \mathbb{Z}_{n}$ singularities. In this scheme one starts with a set of D-branes on $\mathbb{C}^{3}$, arranged according to the regular representation of $\Gamma$, followed by a truncation to the $\Gamma$-invariant sector of the action, thus ending up with a gauged sigma-model whose configuration space, in the low-energy limit, is interpreted as the sub-stringy space-time. This picture has a mathematical counterpart in terms of blowing up a singular Kähler quotient [5, 6] in an orbifold, viewed as a toric variety.

In this note we shall study another example of this construction, namely a blown-up $\mathbb{C}^{3} /\left(\mathbb{Z}_{2} \times \mathbb{Z}_{2}\right)$, following the lines of [3]. We shall find that, in this case, the D-brane probe senses a double-point singularity, which gets resolved for a generic value of the resolution parameter; and under a smooth variation of the resolution parameter one moves on from one resolution to another, which corresponds to a flop [7, 8].

\section{Moduli Space of D-BRanes}

The moduli space of Calabi-Yau compactification has been studied in different ways including either the construction of the mirror manifold and thereby studying its complex structure moduli space [9], or the construction of moduli space of the linear sigma model and subjecting it to the action of a renormalization group in order to yield a conformal-invariant physical space [10,11]. Both approaches yield a moduli space that has regions which either have a geometric interpretation or at least can be connected to a geometric phase by means of an analytic continuation. Since D-branes are natural probes of (sub-stringy) space-time, it is important to study the geometric structure sensed by them.

This kind of study differs from the conventional string theoretic studies in that, while a classical background geometry is incorporated in an ad hoc fashion into string theory, and is then subjected to various corrections, D-brane geometry emerges from the study of the moduli space of the vacuum configuration of D-branes [2]. Thus, at the regime of weak coupling, the geometry could be understood through a classical study of the vacuum of the world-volume moduli space of the corresponding supersymmetric Yang-Mills theory. This world-volume theory turns out to be classically described by a gauged linear sigma model giving rise to space-times avoiding the occurrence of non-geometric phases. This moduli space is likely to admit quantum correction in general, but for D0-branes at weak coupling those corrections are suppressed [3]. Further, if we expect, following [12, and also [3]], that there is no surprise lurking in the strong coupling

Date: July 2, 2018. 
regime, and extrapolate this result to strong coupling, then this is in conformity with the picture that arises in considerations with M-theory [ $\left[\right.$. In this note we shall discuss D-branes on an orbifold of $\mathbb{C}^{3}$ obtained by adding the moduli from the twisted sector of the theory with small coefficients.

$\mathrm{D} p$-branes near an orbifold singularity is represented by a super Yang-Mills Lagrangian with FayetIliopoulos term due to coupling to the closed string twisted sector [1]. It has been shown from world-sheet consistency conditions that the orbifold prescription of the closed string theory also applies to the open string theory, with new open string sectors provided by the images of the original D-brane [13]. Thus a $\mathrm{D} p$-brane at a point in $\mathbb{C}^{3} / \Gamma$ will be defined as the quotient of the theory of $|\Gamma|$ number of $\mathrm{D} p$-branes in $\mathbb{C}^{3}$ by a simultaneous action of $\Gamma$ on $\mathbb{C}^{3}$ and the D-brane index. Here $|\Gamma|$ denotes the dimension of the discrete group $\Gamma$.

Thus, in order to construct the Lagrangian of D-branes compactified on an orbifold $\mathbb{C}^{3} / \Gamma$ of $\mathbb{C}^{3}$, where $\Gamma=\mathbb{Z}_{2} \times \mathbb{Z}_{2}$ is a third order discrete subgroup of $S U(3)$, we start with a theory of four D-branes in $\mathbb{C}^{3}$. In view of the observation that the world-volume theory of D0-branes is the dimensionally reduced ten-dimensional $N=1$ super-Yang-Mills theory, the present one is equivalent to the dimensionally reduced $N=4$ super Yang-mills field theory in four dimensions with gauge group $U(n)$. The branes are arranged in $\mathbb{C}^{3}$ in a $\Gamma$-symmetric fashion to begin with. Any generator of the discrete group $\Gamma$ acts on the space $\mathbb{C}^{3}$, as well as on the Chan-Paton degrees of freedom of the branes, the latter action being in the regular representation. We then quotient out the theory by $\Gamma$ by restricting to the $\Gamma$-invariant sector of the theory.

Let us begin by describing the action of $\Gamma$ on the bosonic fields. The corresponding fermionic partners can be incorporated at any stage of the calculation cashing in on supersymmetry. As there are four D-branes, they are represented by $4 \times 4$ matrices transforming in the adjoint of $U(4)$. For the sake of convenience we write the bosonic fields corresponding to the coordinates tangential to the $\mathbb{C}^{3}$ as a set of three $4 \times 4$ complex matrices, $\left\{X^{1}, X^{2}, X^{3}\right\}$. We shall then quotient them out by the discrete group $\Gamma=\mathbb{Z}_{2} \times \mathbb{Z}_{2}$. Let us denote the three generators of $\mathbb{Z}_{2} \times \mathbb{Z}_{2}$ by $\mathfrak{g}_{1}, \mathfrak{g}_{2}$ and $\mathfrak{g}_{1} \mathfrak{g}_{2}$. The action of the generators $\mathfrak{g}_{1}$ and $\mathfrak{g}_{2}$ on the $X$ 's are given as:

$$
X^{\mu} \longrightarrow R_{\nu}^{\mu} \gamma\left(\mathfrak{g}_{i}\right) X^{\nu} \gamma\left(\mathfrak{g}_{i}\right)^{-1}
$$

where $R_{\nu}^{\mu}$ denotes a rotation matrix associated to the generators, defined as

$$
R_{\nu}^{\mu}\left(\mathfrak{g}_{1}\right)=\operatorname{Diag}\{-1,-1,+1\}, \quad R_{\nu}^{\mu}\left(\mathfrak{g}_{2}\right)=\operatorname{Diag}\{-1,+1,-1\} ;
$$

while we have defined the matrices

$$
\gamma\left(\mathfrak{g}_{1}\right)=\operatorname{Diag}\{1,-1,1,-1\}, \quad \gamma\left(\mathfrak{g}_{2}\right)=\operatorname{Diag}\{1,1,-1,-1\},
$$

with $\gamma$ denoting a regular representation of the corresponding generator written as its argument. For the fields corresponding to the coordinates transverse to the $\mathbb{C}^{3}$ in question, the action is the same except that they are represented by real matrices and the rotation matrix is the identity: $R_{\nu}^{\mu}=\delta_{\nu}^{\mu}$. The fermionic fields are also represented by $4 \times 4$ matrices with a similar action of $\Gamma$ on them.

On quotienting by $\mathbb{Z}_{2} \times \mathbb{Z}_{2}$ with the above-mentioned action, the fields corresponding to the coordinates transverse to $\mathbb{C}^{3}$ become diagonal, while the three fields for the tangential ones assume the following forms:

$$
X^{1}=\left(\begin{array}{cccc}
0 & 0 & 0 & x_{1} \\
0 & 0 & x_{2} & 0 \\
0 & x_{3} & 0 & 0 \\
x_{4} & 0 & 0 & 0
\end{array}\right), \quad X^{2}=\left(\begin{array}{cccc}
0 & y_{1} & 0 & 0 \\
y_{2} & 0 & 0 & 0 \\
0 & 0 & 0 & y_{3} \\
0 & 0 & y_{4} & 0
\end{array}\right), \quad X^{3}=\left(\begin{array}{cccc}
0 & 0 & z_{1} & 0 \\
0 & 0 & 0 & z_{2} \\
z_{3} & 0 & 0 & 0 \\
0 & z_{4} & 0 & 0
\end{array}\right),
$$

where $x_{i}, y_{i}$ and $z_{i}, i=1,2,3,4$, are complex numbers. This leaves a subgroup $U(1)^{4}$ of $U(4)$ unbroken. An overall $U(1)$ aside, we thus have a gauge group $U(1)^{3}$, which is further broken by the non-zero expectation values of the chiral fields, $X^{1}, X^{2}$ and $X^{3}$. Thus, the resulting theory represents a single D-brane in the orbifold, $\mathbb{C}^{3} /\left(\mathbb{Z}_{2} \times \mathbb{Z}_{2}\right)$.

In order to take into account the coupling of the D-brane to the closed string twisted sector of the theory, one needs to introduce Fayet-Iliopoulos terms in the Lagrangian. Since in this case there are three generators of $\Gamma$, we get three real scalars from the NS-NS sector which are known to be responsible for blowing up the orbifold singularity [1,2]. World-sheet considerations [1] indicate that in the presence of a twisted sector the theory picks up Fayet-Iliopoulos terms such that the coefficient $\zeta_{i}$ of the $i$-th Fayet-Iliopoulos term corresponds to the $i$-th of the three $U(1)$ 's and the value of each $\zeta_{i}$ is determined by the expectation value of the NS-NS moduli fields. 
As explained earlier, the space-time sensed by the D-brane can be derived from the moduli space of the above theory. Since we are interested in the supersymmetric vacua of the theory, we impose the D-flatness condition, namely,

$$
\begin{aligned}
& \zeta_{1}=-\left|x_{1}\right|^{2}+\left|x_{4}\right|^{2}-\left|y_{1}\right|^{2}+\left|y_{2}\right|^{2}-\left|z_{1}\right|^{2}+\left|z_{3}\right|^{2}, \\
& \zeta_{2}=-\left|x_{2}\right|^{2}+\left|x_{3}\right|^{2}-\left|y_{2}\right|^{2}+\left|y_{1}\right|^{2}-\left|z_{2}\right|^{2}+\left|z_{4}\right|^{2}, \quad \text { and } \\
& \zeta_{3}=-\left|x_{3}\right|^{2}+\left|x_{2}\right|^{2}-\left|y_{3}\right|^{2}+\left|y_{4}\right|^{2}-\left|z_{3}\right|^{2}+\left|z_{1}\right|^{2} .
\end{aligned}
$$

Let us note that the coefficients $\left\{\zeta_{i} \mid i=1,2,3\right\}$ satisfy the condition $\sum_{i=1}^{3} \zeta_{i}=0$, as is required for the existence of supersymmetric vacua [3].

Let us now consider the superpotential of the theory, which is given by

$$
\mathcal{W}=\operatorname{Tr}\left[X^{1}, X^{2}\right] X^{3}
$$

The vacuum satisfies the following condition,

$$
\begin{array}{r}
\partial \mathcal{W}=0, \quad \text { or equivalently, } \\
{\left[X^{\mu}, X^{\nu}\right]=0 .}
\end{array}
$$

We first evaluate the superpotential constraints. Using the expressions (4) in (9), we derive twelve constraint relations on the twelve variables. However, out of these twelve constraint relations six could be seen to be independent, which we choose as the following ones:

$$
\begin{aligned}
x_{1} x_{4}=x_{2} x_{3}, & x_{1} y_{4}=y_{1} x_{2}, \\
y_{1} y_{2}=y_{3} y_{4}, & x_{1} z_{4}=z_{1} x_{3}, \\
z_{1} z_{3}=z_{2} z_{4}, & y_{1} z_{2}=z_{1} y_{3} .
\end{aligned}
$$

One can derive the other six equations that follow from the superpotential constraint using these six. Thus, we are left with six independent variables from the components of the $X$ 's. The D-brane moduli space is constructed out of these independent components. The classical D-brane moduli space is obtained for a fixed set of values of the Fayet-Iliopoulos coefficients $\zeta_{i}$, by imposing the relations (5)-(7) and the superpotential constraint $(10)$ and further quotienting out by the residual $U(1)^{3}$ gauge group. Let us now describe this quotient construction following [3].

\section{TOPOLOGICAL PROPERTIES}

We want to find out the toric variety that describes the moduli space of D-branes obtained via the quotient construction mentioned above. In other words, we want to describe the space of solutions of the superpotential constraints, after quotienting out by the residual $U(1)^{3}$ gauge group.

In the present case we are dealing with the three-(complex)-dimensional moduli space of the D-branes. This can be described as a quotient space $\left(\mathbb{C}^{9}-\mathfrak{F}_{\Delta}\right) /\left(\mathbb{C}^{\star}\right)^{6}$ for some set $\mathfrak{F}_{\Delta}$ determined by the restricted set of values of $\zeta_{i}, i=1,2,3$, which are relevant in this case and an action of $\left(\mathbb{C}^{\star}\right)^{6}$ on the ambient $\mathbb{C}^{9}$. Different choices of values of $\zeta_{i}, i=1,2,3$ correspond to different phases of the theory, which are related to the set $\mathfrak{F}_{\Delta}$ to be removed from the ambient space $\mathbb{C}^{9}$.

In order to construct a toric variety one needs a lattice and a fan [15], which is a set of strongly convex rational polyhedral cones, $\{\mathfrak{s}\}$. For the case at hand we take the lattice as $\mathfrak{M}=\mathbb{Z}^{6}$ and then choosing the independent variables as $\left\{x_{1}, y_{1}, y_{2}, z_{1}, z_{2}, z_{4}\right\}$, we define the cone $\mathfrak{s}$ as being generated by the the indices of 
the independent variables in the expressions of the twelve variables given in a matrix form as follows:

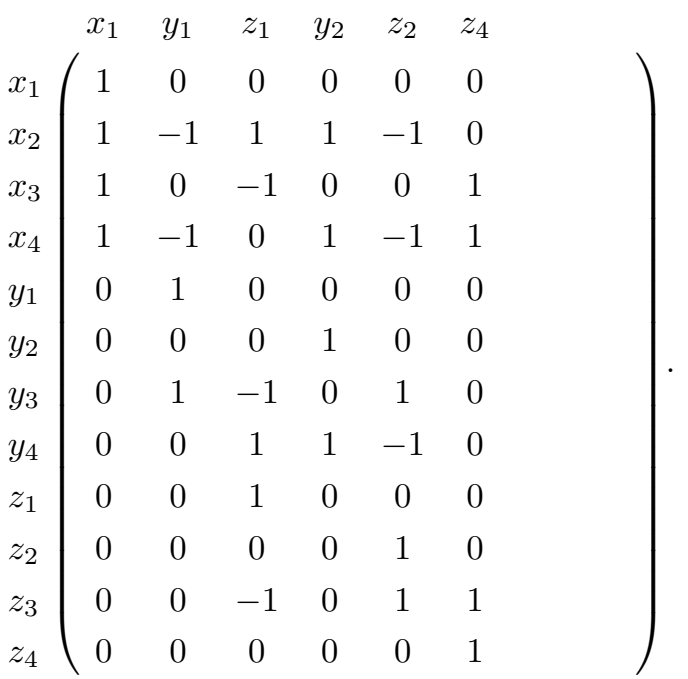

In other words, the cone $\mathfrak{s}$ is generated by the rows of the rectangular $12 \times 6$ matrix. Now to describe the toric variety, it is particularly convenient to consider the dual cone $\mathfrak{s}^{\vee}$. The dual cone is defined in a lattice $\mathfrak{M}^{\vee}=\operatorname{Hom}(\mathfrak{M}, \mathbb{Z})$, dual to $\mathfrak{M}$. One writes the twelve variables $\left\{x_{i}, y_{i}, z_{i} \mid i=1,2,3,4\right\}$ in terms of nine homogeneous coordinates $\left\{p_{i} \mid i=0, \cdots 8\right\}$ [16, also [3]]. Indices of the homogeneous coordinates, as they appear in the monomials defining the original variables, determine the dual cone $\mathfrak{s}^{\vee}$. The columns of the matrix $T$ specifying a map

(12) $T: \mathbb{C}\left[p_{0}, p_{1}, p_{2}, p_{3}, p_{4}, p_{5}, p_{6}, p_{7}, p_{8}\right] \longrightarrow \mathbb{C}\left[x_{1}, y_{1}, y_{2}, z_{1}, z_{2}, z_{4}, x_{1}^{-1}, y_{1}^{-1}, y_{2}^{-1}, z_{1}^{-1}, z_{2}^{-1}, z_{4}^{-1}\right]$ generate the cone $\mathfrak{s}^{\vee}$ :

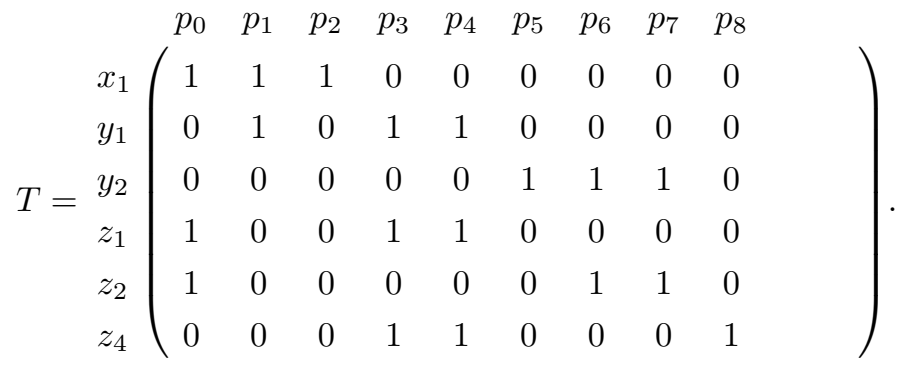

The transpose of the kernel of $T$, which is a $3 \times 9$ matrix, given by

$$
(\operatorname{Ker} T)^{\mathrm{T}}=\left(\begin{array}{lllllllll}
-1 & -1 & 2 & 0 & 1 & -1 & 0 & 1 & -1 \\
-1 & -1 & 2 & 1 & 0 & -1 & 1 & 0 & -1 \\
-1 & -1 & 2 & 1 & 0 & -1 & 0 & 1 & -1
\end{array}\right)
$$

then specifies the action of the algebraic torus $\left(\mathbb{C}^{\star}\right)^{3}$ on $\mathbb{C}^{9}$. However, for our case we have to further take into account the fact that the whole variety thus obtained is not relevant. The relevant part is what is left over after quotienting by the $U(1)^{3}$ arising in the twisted sector of the moduli space. In order to dispense with the irrelevant part, one has to consider the inclusion of an extra $\left(\mathbb{C}^{\star}\right)^{3}$ in $\mathbb{C}^{9}$. As a whole, this is tantamount to prescribing an action of $\left(\mathbb{C}^{\star}\right)^{6}$ on the toric variety. Since the set $\mathbb{C}^{9}-\mathfrak{F}_{\Delta}$ contains $\left(\mathbb{C}^{\star}\right)^{9}$, the toric variety $\left(\mathbb{C}^{9}-\mathfrak{F}_{\Delta}\right) /\left(\mathbb{C}^{\star}\right)^{3}$ contains $\left(\mathbb{C}^{\star}\right)^{9} /\left(\mathbb{C}^{\star}\right)^{3} \simeq\left(\mathbb{C}^{\star}\right)^{6}$ as a dense open subset. As a group this acts on the toric variety through an action of $\left(\mathbb{C}^{\star}\right)^{6}$ on $\mathbb{C}^{9}$, provided the projection of the action to $\left(\mathbb{C}^{\star}\right)^{9} /\left(\mathbb{C}^{\star}\right)^{3} \simeq\left(\mathbb{C}^{\star}\right)^{6}$ gives the identity map. Such an action is specified by a $6 \times 9$ charge matrix, denoted $U$, and the condition on the projection is,

$$
T U^{\mathrm{T}}=\mathbb{I}_{6},
$$

where $T$ is the $6 \times 9$ matrix defined in (13). In order to find out the matrix $U$, we thus have to invert the rectangular matrix $T$. We use the Moore-Penrose prescription which gives a unique inverse. The matrix $U^{\mathrm{T}}$ 
is evaluated as the transpose of the Moore-Penrose inverse of the transpose of $T$. It takes the following form:

$$
U=\left(\begin{array}{ccccccccc}
2 / 9 & 2 / 9 & 5 / 9 & -1 / 9 & -1 / 9 & 2 / 9 & -1 / 9 & -1 / 9 & 2 / 9 \\
-1 / 3 & 2 / 3 & -1 / 3 & 1 / 6 & 1 / 6 & -1 / 3 & 1 / 6 & 1 / 6 & -1 / 3 \\
-1 / 9 & -1 / 9 & 2 / 9 & 1 / 18 & 1 / 18 & 8 / 9 & 1 / 18 & 1 / 18 & -1 / 9 \\
1 / 2 & -1 / 2 & 0 & 1 / 4 & 1 / 4 & 1 / 2 & -1 / 4 & -1 / 4 & -1 / 2 \\
1 / 6 & 1 / 6 & -1 / 3 & -1 / 12 & -1 / 12 & -5 / 6 & 5 / 12 & 5 / 12 & 1 / 6 \\
-1 / 9 & -1 / 9 & 2 / 9 & 1 / 18 & 1 / 18 & -1 / 9 & 1 / 18 & 1 / 18 & 8 / 9
\end{array}\right) .
$$

In order to describe the (symplectic or holomorphic) quotient of $\mathbb{C}^{9}$ with respect to $U(1)^{3}$, that is $\mathbb{C}^{9} / / U(1)^{3}$, we need specify the charges through which the independent fields couple to the three $U(1)$ gauge fields associated with the three Fayet-Iliopoulos terms. These are given by

$$
V=\left(\begin{array}{cccccc}
-1 & -1 & 1 & -1 & 0 & 0 \\
0 & 1 & -1 & 0 & -1 & 1 \\
0 & 0 & 0 & 1 & 0 & 0
\end{array}\right)
$$

Then multiplying $V$ and $U$ one derives,

$$
V U=\left(\begin{array}{ccccccccc}
-1 / 2 & -1 / 2 & 0 & -1 / 4 & -1 / 4 & 1 / 2 & 1 / 4 & 1 / 4 & 1 / 2 \\
-1 / 2 & 1 / 2 & 0 & 1 / 4 & 1 / 4 & -1 / 2 & -1 / 4 & -1 / 4 & 1 / 2 \\
1 / 2 & -1 / 2 & 0 & 1 / 4 & 1 / 4 & 1 / 2 & -1 / 4 & -1 / 4 & -1 / 2
\end{array}\right) .
$$

Multiplying $V U$ with an innocuous factor of 4 and then concatenating with the charge matrix (14) one gets, after some row-operations, the following matrix describing the embedding of the algebraic torus $\left(\mathbb{C}^{\star}\right)^{6}$ in $\mathbb{C}^{9}$.

$$
\widetilde{Q}=\left(\begin{array}{ccccccccc}
-1 & -1 & 2 & 0 & 1 & -1 & 0 & 1 & -1 \\
-1 & -1 & 2 & 1 & 0 & -1 & 1 & 0 & -1 \\
-1 & -1 & 2 & 1 & 0 & -1 & 0 & 1 & -1 \\
-2 & -2 & 0 & -1 & -1 & 2 & 1 & 1 & 2 \\
-2 & 2 & 0 & 1 & 1 & -2 & -1 & -1 & 2 \\
2 & -2 & 0 & 1 & 1 & 2 & -1 & -1 & -2
\end{array}\right)
$$

The co-kernel of the transpose of $\widetilde{Q}$, after ignoring the repetitions, takes the following form:

$$
\widetilde{T}=\operatorname{coKer} \widetilde{Q}^{\mathrm{T}}=\left(\begin{array}{cccc}
0 & 0 & 1 & -1 \\
0 & 1 & 0 & 1 \\
1 & 0 & 0 & 1
\end{array}\right)
$$

and further, one can obtain the kernel of $\widetilde{T}$ to be:

$$
\text { Ker } \widetilde{T}=\left(\begin{array}{c}
-1 \\
-1 \\
1 \\
1
\end{array}\right)
$$

Using (21) one can define the semigroup $\mathfrak{S}_{\mathfrak{s}}$ generated finitely by the toric ideal $\langle x y-z w\rangle$ 15. The corresponding "group" ring can be written as

$$
\mathbb{C}\left[\mathfrak{S}_{\mathfrak{s}}\right]=\mathbb{C}[x, y, z, w] /\langle x y-z w\rangle ;
$$

and therefore one derives,

$$
\text { Spec } \mathbb{C}\left[\mathfrak{S}_{\mathfrak{s}}\right]=x y-z w,
$$

where $x, y, z, w$ are four complex numbers. Recalling that the equation of a conifold in $\mathbb{C}^{4}$ can be cast in the form [17, for example]

$$
x y-z w=0, \quad \text { with } x, y, z, w \in \mathbb{C},
$$

one concludes that the toric variety defined by $\widetilde{T}$ is the blow-up of the conifold (24). Blow-up of the conifold (24) has been studied in detail in 17.

The above moduli space can also be realized in terms of the alternative description of the gauged linear sigma model due to Witten. The homogeneous variables $\left\{p_{i}\right\}$ play the role of the chiral superfields which are charged with respect to the gauge group $U(1)^{3}$. The charge of the $i$-th field with respect to the $j$-th $U(1)$ 
is given by the entry $\widetilde{Q}_{i j}$ of the charge matrix (19). The Fayet-Iliopoulos parameters are again given by $\zeta_{i}$ associated only with the $U(1)$ 's coming from the original world volume theory. The coefficients corresponding to the other $U(1)$ 's vanish. The moduli space again follows from the D-term equation corresponding to this model. This is known as symplectic quotienting [10, 11], where one views $\mathbb{C}^{\star}$ as $\mathbb{R}_{+} \times U(1)$. Apart from imposing the D-term equation, one has to again mod out the remaining three $U(1)$ 's to get the moduli space, as before.

In order to describe the moduli space in a simple form, let us start with the charge matrix $\widetilde{Q}$ with the Fayet-Iliopoulos parameters adjoined to it. So the matrix becomes

$$
\widetilde{Q}=\left(\begin{array}{cccccccccc}
-1 & -1 & 2 & 0 & 1 & -1 & 0 & 1 & -1 & 0 \\
-1 & -1 & 2 & 1 & 0 & -1 & 1 & 0 & -1 & 0 \\
-1 & -1 & 2 & 1 & 0 & -1 & 0 & 1 & -1 & 0 \\
-2 & -2 & 0 & -1 & -1 & 2 & 1 & 1 & 2 & \zeta_{1} \\
-2 & 2 & 0 & 1 & 1 & -2 & -1 & -1 & 2 & \zeta_{2} \\
2 & -2 & 0 & 1 & 1 & 2 & -1 & -1 & -2 & \zeta_{3}
\end{array}\right)
$$

After some row-operations we obtain,

$$
\widetilde{Q}=\left(\begin{array}{cccccccccc}
0 & 0 & 0 & 0 & -2 & 0 & 0 & +2 & 0 & -\frac{\zeta_{2}+\zeta_{3}}{2} \\
0 & 0 & 0 & 0 & 0 & 0 & 1 & -1 & 0 & 0 \\
0 & 0 & 0 & 1 & -1 & 0 & 0 & 0 & 0 & \frac{\zeta_{2}+\zeta_{3}}{4} \\
0 & 0 & -4 & 0 & 0 & 4 & 0 & -4 & 4 & \zeta_{1}+\zeta_{2}+\zeta_{3} \\
-4 & 0 & 0 & 0 & 0 & 0 & 0 & 0 & 4 & \zeta_{1}+\zeta_{2} \\
0 & -4 & 0 & 0 & 0 & 4 & 0 & 0 & 0 & \zeta_{1}+\zeta_{3}
\end{array}\right) .
$$

The equations ensuing from all the rows of the matrix $\widetilde{Q}$, except the fourth one, merely express one variable in terms of the others. Hence these do not impose any non-trivial constraint on the resulting space. One can just trade one variable for the other and thus, finally, the independent variables to consider reduces to $p_{2}, p_{5}, p_{7}$ and $p_{8}$. They are constrained by the non-trivial equation given by the fourth row which is,

$$
\left|p_{2}\right|^{2}-\left|p_{5}\right|^{2}+\left|p_{7}\right|^{2}-\left|p_{8}\right|^{2}=-\frac{\left(\zeta_{1}+\zeta_{2}+\zeta_{3}\right)}{4} .
$$

When all the moduli parameters vanish, this becomes a conifold with a node, while for any generic value the node gets resolved into a $\mathcal{O}(-1) \times \mathcal{O}(-1)$-bundle over the one-dimensional projective space $\mathbb{P}^{1}$. So crossing the singularity corresponds to a flop transition.

\section{Discussion}

In this note we have considered certain topological properties of D-brane moduli space near an Abelian orbifold singularity, $\mathbb{C}^{3} /\left(\mathbb{Z}_{2} \times \mathbb{Z}_{2}\right)$. We have found that the moduli describe a space with a resolved doublepoint singularity. The Fayet-Iliopoulos parameters originating in the closed string twisted sector control the size of the blow-up.

In this analysis we have taken the metric to be flat. We certainly wish that the final metric on the blownup conifold were Ricci-flat and matches the one that can be obtained from low-energy classical supergravity considerations, valid for small values of the curvature, i.e. $R<<1 / l_{p}^{2}$, where $l_{p}$ denotes the Planck length. However, as is the case with $\mathbb{Z}_{3}$ or $\mathbb{Z}_{5}[3]$, it might happen in certain cases that the metric on the resolution is not Ricci flat: perhaps only the bound states of a large number of D-branes will sense a Ricci flat metric [3]. Therefore, it would be interesting to investigate whether in the present case the metric on the resolved conifold is Ricci flat or not. Such a metric has been found earlier in studying conifolds [17]. Work in this direction is in progress.

In the present example we have got the resolutions which are connected by flops. A similar construction for the drastic conifold transition with D-branes may help understanding the topology changing process from the short-distance viewpoint.

It would also be interesting to generalize these considerations for the $D_{n}$ and $E_{n}$ series of groups. At the moment it is not clear whether for these non-Abelian groups one can even visualize the moduli space as a toric variety. If $\Gamma$ does not belong to $S U(3)$, then the resulting theory will be a non-supersymmetric theory and it may be interesting to investigate those theories too. 
Note Added: After completion of this work two related papers appeared [18, 19]. One of these [19] has overlap with ours.

Acknowledgement: It is a pleasure to thank A. Kumar for stimulating discussions and useful suggestions.

\section{REFERENCES}

[1] M. Douglas and G. Moore D-Branes, Quivers, and ALE Instantons; hep-th/9603167.

[2] C. Johnson and R. Myers Aspects of Type IIB Theory on ALE Spaces; hep-th/9610140.

[3] M. Douglas, B. Greene and D. Morrison Orbifold Resolution by D-Branes; hep-th/9704151.

[4] E. Witten Phase Transitions in M theory and F theory; hep-th/9603150, Nucl. Phys. B471 (1996) 195.

[5] A. Sardo Infirri Partial Resolutions of Orbifold Singularities via Moduli Spaces of HYM-type Bundles; alg-geom/9610004.

[6] A. Sardo Infirri Resolutions of Orbifold Singularities and Flows on the McKay Quiver; alg-geom/9610005.

[7] P. Candelas, B. Greene and T. Hubsch Rolling among Calabi-Yau Vacua; Nucl. Phys. B330 (1990) 49.

[8] B. Greene String theory on Calabi-Yau Manifolds; hep-th/9702155.

[9] P. Aspinwall, B. Greene and D. Morrison Calabi-Yau moduli space, mirror manifolds and space-time topology change in string theory; hep-th/9309097, Nucl. Phys. B416 (1994) 414.

[10] E. Witten Phases of $N=2$ theories in Two Dimensions; hep-th/9301042, Nucl. Phys. B403 (1993) 159.

[11] P. Aspinwall and B. Greene On the geometric interpretation of $N=2$ superconformal theories; Nucl. Phys. B437 (1995) 205.

[12] T. Banks, W. Fischler, S. Shenker and L. Susskind M Theory As A Matrix Model: A Conjecture; hep-th/9612126, Phys. Rev. D403 (1993) 159.

[13] E. Gimon and J. Polchinski Consistency Conditions for Orientifolds and D-Manifolds; hep-th/9601038, Phys. Rev. D54 (1996) 1667.

[14] M. Douglas, H. Ooguri and S. Shenker Issues in M(atrix) Theory Compactification; hep-th/9702203.

[15] W. Fulton Introduction to Toric Varieties; Princeton University Press, 1993.

[16] D. Cox Recent developments in toric geometry; alg-geom/9606016.

[17] P. Candelas and X. de la Ossa Comments on Conifolds; Nucl. Phys. B342 (1990) 246.

[18] T. Muto D-branes on Orbifolds and Topology Change; hep-th/9711090.

[19] B. Greene D-brane topology changing transitions; hep-th/9711124.

(Subir Mukhopadhyay) Institute of Physics, Bhubaneswar 751 005, India

E-mail address: subir@iopb.stpbh.soft.net

(Koushik Ray) INFN - Sezione di Roma, "Tor Vergata", Via della Ricerca Scientifica, 100133 Roma, ITAly

E-mail address: koushik@roma2.infn.it 